\title{
Spatial Mapping of Morphology and Electronic Properties of Air-Printed Pentacene Thin Films
}

\author{
Shaurjo Biswas, Yongsoo Yang, Christian M. Schlepütz, Nadav Geva, \\ Randall L. Headrick, Ron Pindak, Roy Clarke, and Max Shtein**
}

To accelerate the pace of materials discovery and application, comprehensive links need to be established between a material's structure, properties, and process conditions used to obtain the material and/or final application format. This work examines the dry printing of pentacene thin film transistor (TFT) channels by guard flow-enhanced organic vapor jet printing (GF-OVJP), a technique that enables direct, solvent-free, additive patterning of devicequality molecular semiconductors in air. Deposition in air entails non-trivial effects at the boundary between ambient surroundings and the gas jet carrying the semiconductor vapor that influence the morphology and properties of the resulting electronic devices. Synchrotron X-ray diffraction is employed, complemented by measurement of electronic properties of GF-OVJP deposited films in a TFT to reveal how the morphology and electronic properties of the films depend on thickness, location within the printed pattern, nozzle translation velocity, and other process parameters. The hole field-effect mobility of the printed pentacene film is linked quantitatively with its crystallinity, as well as with extent of exposure to ambient air during deposition. The analysis can be extended to accurately predict the performance of devices deposited in air by GF-OVJP, which are demonstrated here for a planar, large area deposit.

\section{Background and Motivation}

Increasingly, the development and application of functional materials aims to predict the structure and properties of materials prior to synthesis or processing. ${ }^{[1]}$ While well-developed in metallurgy, polymer synthesis and, to some extent, in silicon processing, relatively little predictive ability exists in the rapidly growing field of organic semiconductors, ${ }^{[2,3]}$ due to the relative novelty of the materials and corresponding processing methods. The present study aims to address this knowledge gap.

Interest in organic-based electronic and optoelectronic devices continues to grow, accompanied by improvements in device performance, ${ }^{[4,5]}$ and commercialization efforts. In particular, organic field effect transistors using conjugated oligoacenes (e.g., pentacene) ${ }^{[6]}$ have exhibited robust performance characteristics, while offering low material cost, low-temperature processing, and compatibility with flexible substrates as advantages for a variety of applications. ${ }^{[7-9]}$

The properties of one archetypal oligoacene, pentacene, have been studied extensively. ${ }^{[6,9-18]}$ The electronic characteristics of pentacene films depend on purity, molecular ordering and film morphology, ${ }^{[6,10]}$ which are in turn affected by process conditions. Pentacene films exhibit polymorphism, which have been extensively studied with respect to their structure, structureprocess and structure-property relationships. ${ }^{[1,12]}$ The films are typically polycrystalline, and it is generally believed that carrier transport improves in films exhibiting well-ordered domains, and in channels with fewest grain boundaries. ${ }^{[13,14]}$ Many attempts have been made to improve carrier transport in the films by increasing grain size ${ }^{[15]}$ or reducing the channel length to approach the size of individual grains. ${ }^{[16]}$ Additionally, improvements in electronic performance characteristics have been realized via material purification, ${ }^{[6]}$ and substrate modification at the interface between the organic semiconductor and the gate dielectric, ${ }^{[14,17,18]}$ and/or between the organic semiconductor and the source/drain contacts. ${ }^{[19]}$

Concomitant with the development of materials and device architectures, there has been considerable effort underway 
to develop better processing methods. Specifically, there is interest in realizing solvent-free deposition and patterning, to minimize unintentional doping of the organic semiconductor by residual solvent, and to reduce process complexity, while minimizing environmental impact. Ideally, the desired semiconductor channel pattern could be generated rapidly in a single step, at ambient conditions, directly on the substrate of interest, with minimal material waste. None of the solvent-free deposition methods reported to date (including vacuum-based thermal evaporation (VTE), ${ }^{[20]}$ organic vapor phase deposition (OVPD), ${ }^{[21]}$ flash evaporation, ${ }^{[22]}$ laserinduced thermal imaging, ${ }^{[23]}$ early variants of molecular jet printing, ${ }^{[24]}$ and organic vapor jet printing $(\mathrm{OVJP})^{[25,26]}$ satisfy these desired criteria fully. Molecular jet printing and OVJP enable additive patterning without the use of shadow masks. A new variant of OVJP-guard flow-enhanced OVJP-enables further control over the deposited film crystallinity, ${ }^{[27]}$ and can be modified to enable deposition in air. This work tightly links process conditions with film morhpology measured by synchrotron X-ray diffraction, and quantitatively links process conditions and morphology to the film's performance in a TFT.

In OVJP, the organic semiconductor is evaporated into a stream of inert carrier gas, jetted from a nozzle at high velocity onto a cold substrate, where the organic material selectively condenses. Flow and material parameters, apparatus dimensions, and carrier gas determine the resolution of the deposited pattern. When depositing in air, oxygen and moisture from the ambient can be expected to diffuse into the periphery of the vapor jet and potentially modify the properties of the deposited film. In $\mathrm{Alq}_{3}$ deposition, for example, the effects of oxidation have been well studied. ${ }^{28]}$ To minimize oxidation, the organic-containing jet can be surrounded by a guard jet of inert gas. The shielding and hydrodynamic focusing effects of the latter in guard flow-enhanced OVJP (GF-OVJP) growth of $\mathrm{Alq}_{3}$ have been studied, ${ }^{[29]}$ but no report was given with respect to morphological changes with guard flow rate. A subsequent study on the GF-OVJP growth of subphthalocyanine chloride (SubPc) for organic photovoltaic device applications revealed that film morphology can be affected greatly by the guard flow, potentially enhancing cross-plane carrier transport/exciton and photovoltaic device performance, ${ }^{[27]}$ although no report was given regarding effects on in-plane transport.

On the one hand, in the above-mentioned initial studies of GF-OVJP intriguing processing advantages were introduced, including solvent free, rapid, direct, additive, single-step active layer patterning, scalability to large area substrates, no need for drying/annealing steps to obtain desired morphology, ability to deposit multilayered and mixed layers structures, high material utilization efficiency, and ability to grow device-quality films in air. On the other hand, to realize these possibilities, several important questions should be addressed, including: when depositing in air, how much oxygen and moisture is incorporated into the film? What effect/interplay exists between the degree of air exposure, molecular flux, flow conditions, morphology of the deposited film and the resulting device performance? Answering these questions is the basis for studying the deposition technique in detail and doing morphology and structure characterization coupled with flow dynamics simulation and TFT measurements.

In this study, pentacene thin films are grown by GF-OVJP, while studying the morphology based on location within the deposit using ex situ synchrotron X-ray diffraction (XRD); subsequently the electronic properties of the films are characterized and correlated with the deposition conditions and film morphology. As a result of these detailed studies, we propose an analytical expression for the expected in-plane charge mobility in an organic semiconductor film deposited in air by GF-OVJP when rastering the jet over the substrate for large area applications.

\section{Results and Discussion}

The deposition apparatus (Figure 1) consists of two cylindrical, concentric nozzles for the carrier gas and guard flow jets. The annular channel is angled at $15^{\circ}$ from the nozzle axis, shielding and focusing the inner nozzle jet. The process parameters include: nozzle-substrate separation, s, evaporation (sublimation) zone temperature, $T_{\mathrm{Ev}}$, substrate temperature, $T_{\mathrm{S}}$, carrier gas flow rate, $Q_{\mathrm{CG}}$ and guard flow rate, $Q_{\mathrm{GF}}$. Pentacene powder is loaded in the sublimation zone and heated to $T_{\mathrm{Ev}}$ while $Q_{\mathrm{CG}}$ and $Q_{\mathrm{GF}}$ are held constant. Processing conditions are detailed further in the experimental section. When all upstream conditions, including $T_{\mathrm{Ev}}, T_{\mathrm{S}}, Q_{\mathrm{CG}}$, and $Q_{\mathrm{GF}}$ are fixed, the organic vapor flux exiting the nozzle is constant, proportional to the vapor pressure ${ }^{[30]}$ and has cylindrical symmetry. When the nozzle and substrate are stationary relative to each other, at a given value of $s$, a "spot deposit" is obtained which has a bellshaped cross-sectional profile as shown in Figure 1d, with the feature thickness of the spot being determined by the nozzle dwell time, as verified by optical interferometry ${ }^{[31]}$ and FLUENT 6.3.26 hydrodynamic simulations. The profiles of deposits of different thickness (i.e., different nozzle dwell time) have the exact same shape, and all plots of the profiles are normalized to unity.

Moving the nozzle relative to the substrate at a speed $v$, we obtain "line deposits". Line thicknesses obtained by translating the nozzle are linearly proportional to $1 / v$. The thickness of the line deposit is uniform in the direction of motion, while the cross-section thickness profile in the direction perpendicular to motion remains that of a spot deposit. The radial axis in spot deposits and the direction perpendicular to the nozzle translation axis are collinear, denoted as the $r$-axis throughout the text. Similarly, the direction of nozzle motion is denoted as the $x$-axis. Moving the nozzle in multiple passes in a single line can increase the overall deposit thickness; for example, a $60 \mathrm{~nm}$ feature center thickness can be obtained by 4 passes of the nozzle, with each pass contributing $15 \mathrm{~nm}$ to the overall feature height. Note that these "stack" deposits are expected to exhibit different device properties from deposits of the same thickness grown in a single pass, because a "stack" deposit grown in multiple passes is exposed to air a number of times as the nozzle moves back and forth, resulting in a regrowth condition. Rastering the nozzle to draw multiple parallel, adjacent lines with sufficiently narrow line spacing (e.g., $0.1 \mathrm{~mm}$ ) allow the deposit profiles to overlap and form one 
(a)

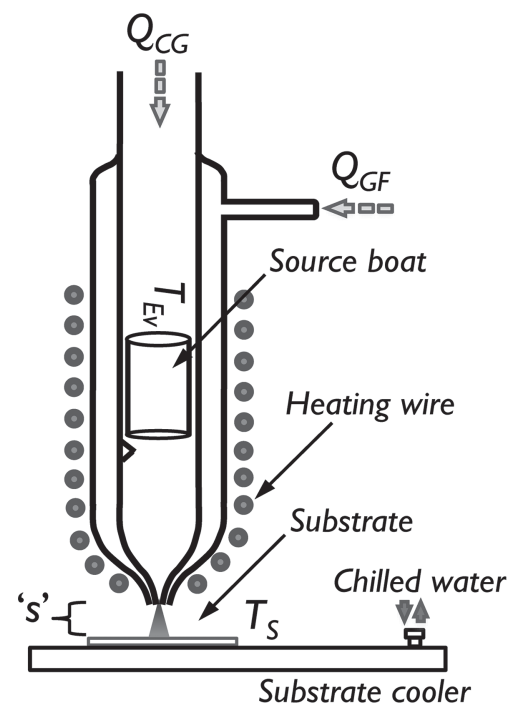

(b)

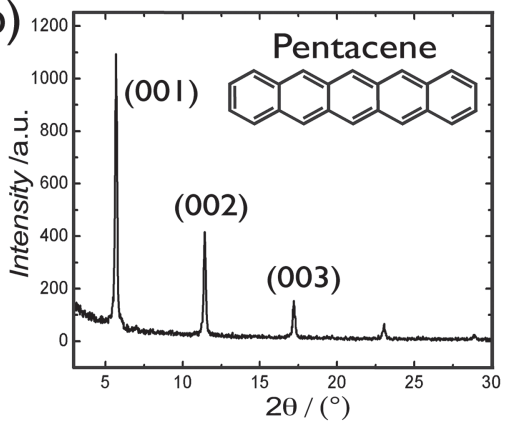

(c)

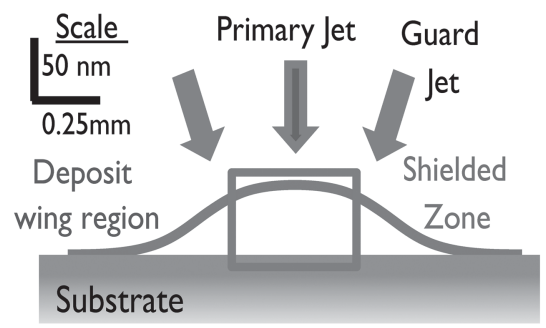

(d)

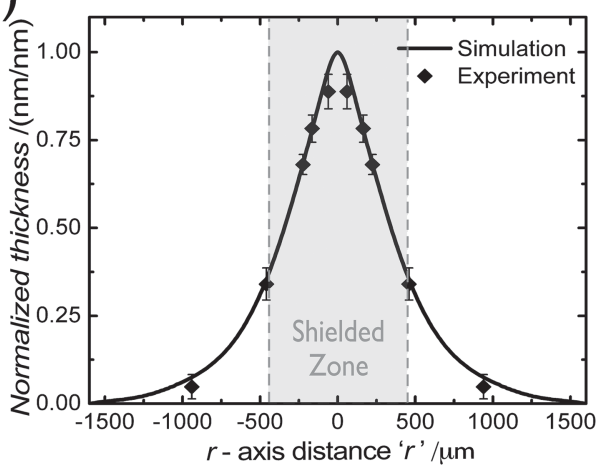

(e)

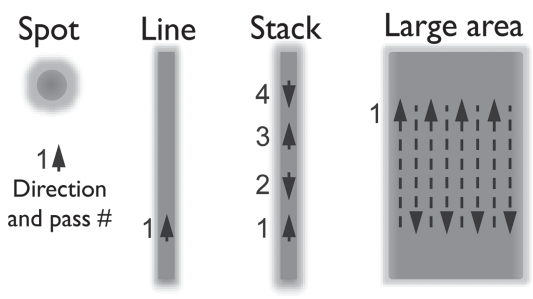

Figure 1. a) Schematic of the GF-OVJP apparatus, indicating the independently variable processing parameters. b) Bragg peaks of a 60 nm thick pentacene film deposited in air by GF-OVJP. The 001 peak measured at $2 \theta=5.7^{\circ}$ using a Cu-K $\alpha$ source indicated the deposit is in thin film phase with a lattice spacing of $1.54 \mathrm{~nm}$. c) Qualitative cross-section (not to scale) of a typical deposition profile, with the central region of the deposit being shielded from ambient oxygen and moisture by the inert guard flow jet. d) GF-OVJP deposits have the same general shape and can be normalized to the profile shown here. This cross-sectional shape is validated by fluid dynamic simulations and by optical interferometric measurements on the deposits. e) Top view of different deposit types obtainable by GF-OVJP by varying the nozzle's translational velocity, number of passes and direction of motion.

"large area deposit" with uniform thickness throughout. ${ }^{[32]}$ Figure 1e schematically depicts the types of deposits described above. In this work, we use all four types of deposits to fully characterize how processing affects the thin film morphology and associated device performance. To fabricate a top (source/ drain) contact TFT on a spot deposit, gold source and drain electrodes can be prepared by VTE through a precisely placed shadow mask. For line deposits, we can deposit gold contacts with the channel width parallel to the direction of motion and hence measure electronic transport across a uniform thickness of film.

The Results section is divided into three sub-sections. First, we analyze the effect of deposition in air on the polycrystalline morphology of the grown films. Then, we study the electronic properties of thin-film pentacene grown in air and in a nitrogen-purged glovebox by GF-OVJP and compare them to TFTs fabricated by VTE. Finally, we isolate the degradation of organic thin films due to oxygen and moisture exposure during deposition, and formulate a simple analytical expression predicting the field-effect mobility of a large-area deposit formed in air by GF-OVJP.

\subsection{Molecular Ordering as a Function of Molecular Flux and Exposure to the Ambient: the Significance of Location Within the Deposited Feature}

In GF-OVJP, the dependence of the deposited thickness on the local molecular flux at the substrate and nozzle translation velocity is non-trivial. In our experiment, the nozzle geometry is held constant and so is the net flux of molecules exiting the nozzle. As mentioned previously, for line deposits, the $r$-axis is the direction perpendicular to nozzle translation direction, $\boldsymbol{x}$. Figure 1d shows that the thickness and molecular flux crosssectional profile vary in the $r$-axis direction. For a given point along the $r$-axis, the thickness of the material after a single pass of the nozzle $\left(t_{\text {pass }}, n m\right)$ depends inversely on the nozzle translation velocity, $v$. The two parameters of interest are the molecular flux (varying along $r$-axis) and the nozzle translational velocity, $v$ (which can be varied along the translation direction); they need to be deconvoluted to examine their effects on the film crystallinity.

First, we look at the effect of $v$ on the crystallinity of the deposit at $r=0 \mathrm{~mm}$ (center of the shielded zone), where the 
molecular flux is constant. Here, the film crystallinity depends primarily on thickness, which in-turn is controlled by $v$. This is discussed in Section 2.1.1 and the synchrotron XRD apparatus details are provided the Section 4.3. Next, we analyze the effect of the positional change on the molecular flux, and the associated $t_{\text {pass }}$, along the $r$-axis $(r>>0)$ for a single pass of the nozzle translated at a particular value of $v$. In this case, the crystallinity is affected not only by the thickness but also by increasing exposure to air along the $r$-axis. This effect is discussed in Section 2.1.2.

\subsubsection{Effect of Nozzle Velocity on the Crystallinity of Pentacene Films}

Any desired deposited feature thickness can be attained either by growing the film at some value of $v$ in one pass, that is, continuous film growth, or at a higher $v$ in multiple passes, that is, intermittent film growth. An important question is: will the crystallinity of the deposited film be identical in the two approaches?

To address this question, ex situ synchrotron X-ray diffraction experiments were performed on the films grown with various numbers of passes for several nozzle velocities (v), while looking at film only at $r=0 \mathrm{~mm}$ of the line deposit. Structural studies of organic thin films deposited in air using X-ray diffraction were carried out at the National Synchrotron Light Source, Brookhaven National Laboratory. The diffraction signal shapes were recorded by a Pilatus $100 \mathrm{~K}$ area detector. Figure 2 shows the intensity at the position of the 001 pentacene film Bragg peak $\left(I_{r=0}\right)$. Four solid lines are shown, with each line representing a particular $v$. For each $v$, different film thicknesses along the corresponding line are obtained by varying

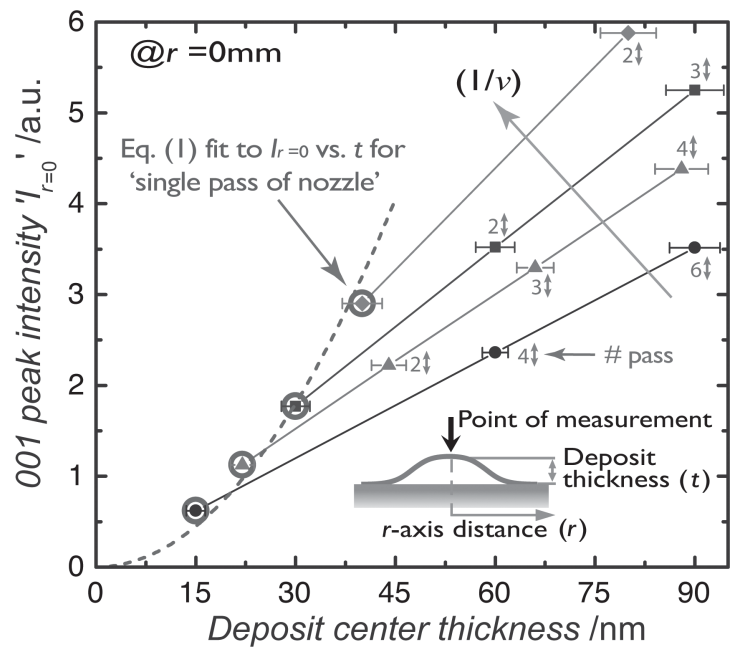

Figure 2. Diffraction intensity at the 001 Bragg peak position is plotted as a function of film thickness (at $r=0 \mathrm{~mm}$ ). The dashed line is a quadratic fit of the intensity measured for films deposited by a single pass of the nozzle (marked with $\bigcirc$ ). Each solid line corresponds to a particular nozzle translation velocity, $v$, and different data points on each line are obtained by multiple passes (numerical indicates the number of passes) of the nozzle at each $v$. the number of passes, with the first point representing a single pass.

As typical for single crystal films, the diffraction intensity at the Bragg peak position increases as a quadratic function of thickness, because the diffracted waves add up coherently. ${ }^{[33]}$ In Figure 2, the first data points on each solid line, with least thickness (marked with $\bigcirc$ ), represent the diffraction intensity from the films deposited with a single pass for each $v$. These data points fit well with the quadratic model (dashed curve). Since these films were grown continuously by a single pass of the nozzle, most of the film would crystallize simultaneously and form a continuous, stacked crystal (defined here as "coherent" crystal), which explains the quadratic behavior, given by

$$
I_{r=0}=0.0019\left(t_{\text {pass }}\right)^{2}
$$

Multiple pass deposition, however, shows a significantly different behavior. As the film thickness is increased by multiple passes of the nozzle at a particular value of $v$, the XRD peak intensity scales linearly with the thickness (solid lines, Figure 2). This indicates that the crystal layers grown by each pass are discontinuous in the out-of-plane direction forming multiple stacked layers of identical "coherent" crystal structures with domain boundaries between the layers. From the standpoint of interaction with the X-rays, these layers diffract as separate crystallites, and the maximum intensities at the Bragg peak position add up linearly. Smaller values of $v$ result in a thicker 'coherent' layer for each pass of the nozzle and thus yield a larger slope of the solid line in Figure 2. This suggests that the films having identical thickness can have significantly different crystallinity, depending on the relative nozzle-substrate motion speed: low $v$ in one pass, or at relatively higher $v$ in multiple passes. This feature is potentially useful, such as when attempting to deposit amorphous films (e.g., emission layers in OLEDs) or crystalline films (e.g., transport layers in OLEDs), depending on the desired functionality.

\section{1.2. Effect of Location Along $\boldsymbol{r}$-Axis and Ambient Exposure on Film Crystallinity}

The design of the nozzle, or the required precision of pattern alignment, is informed by the potential variation of properties across the area of a deposit. When depositing films for TFT or OPV applications, greater crystallinity and purity are generally desirable. For a single pass of the nozzle at a given translation velocity (at any point along $r$-axis) the 001 Bragg peak intensity would scale only with thickness in the absence of ambient air, with the same quadratic relationship (Equation 1 and Figure 2). However, this is not the observed behavior, potentially due to manner in which the guard-flow behaves near the jet's periphery, explored further below.

The jet velocity contours (Figure 3a), temperature and pressure distribution, extent of air incorporation (Figure $3 \mathrm{~b}$ ), and other parameters can be quantified and understood using the fluid dynamics simulation of the GF-OVJP process. As shown in Figure $3 \mathrm{~b}$ for the apparatus geometry used here, the shielded zone provided by the guard flow jet extends to $r=0.45 \mathrm{~mm}$ out 
(a)

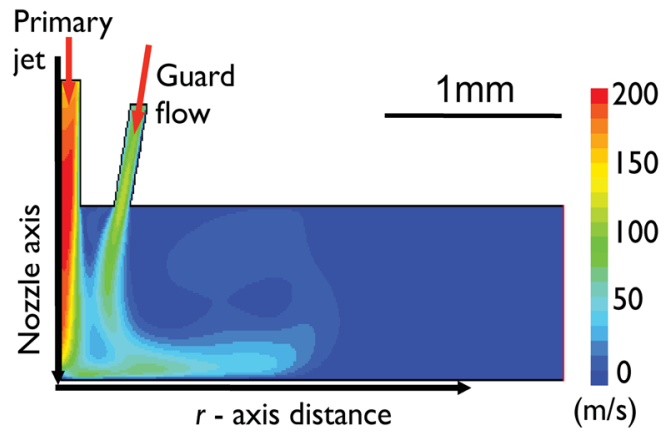

(c)

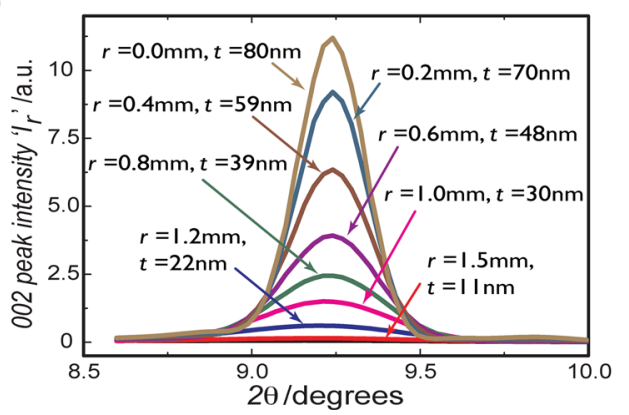

(b)

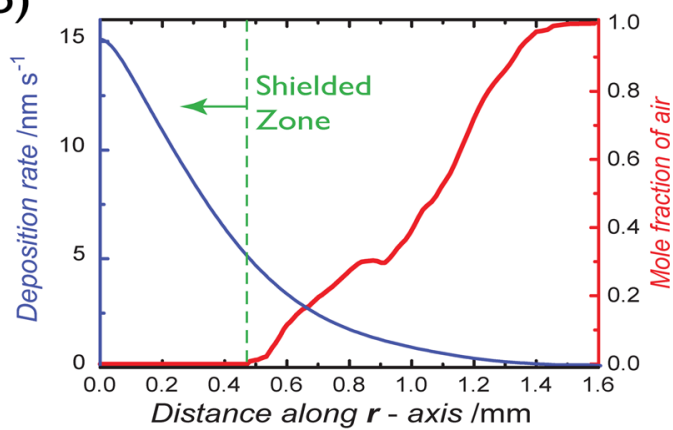

(d)

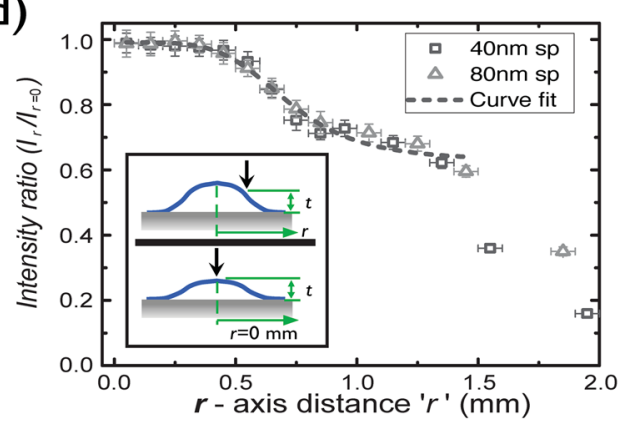

Figure 3. a) Velocity contours obtained from the fluid dynamics simulation of the GF-OVJP process. b) Plot of the simulated mole fraction of air interacting with the hot pentacene molecules during deposition as a function of $r$. c) $\theta-2 \theta$ diffraction intensity, $I_{\mathrm{r}}$, measurements around 002 peak for a line deposit, with feature center thickness of $80 \mathrm{~nm}$, at different $r$-axis locations ( $r$ ) where the thickness is $t$. $d$ ) Ratio of $I_{\mathrm{r}}$ [measured intensities at 002 peak position: peak intensities given in (c)] and $I_{r=0}$, [calculated for corresponding thickness from Equation 1] are plotted for $40 \mathrm{~nm}$ and $80 \mathrm{~nm}$ feature center thickness. Dashed line is a curve fit to the data with Equation 2.

for the deposition conditions mentioned above. The "wing" of the deposit lies beyond the shielded zone $(0.45<r<1.8 \mathrm{~mm})$ and is essentially unprotected by the guard flow. Hence, we expect some degradation in this "wing", since incorporation of oxygen and moisture into the film during deposition results in more defects, both chemical and crystallographic.

Here, the question is: how large of an effect will greater air exposure in the wings of the deposit have on the resulting film structure and properties, compared to when the film has minimal air exposure? We study this question by looking at the diffraction intensity at different points along the $r$-axis (Figure 3c). The intensities, however, cannot be directly compared to each other because both film thickness and air exposure change as a function of $\boldsymbol{r}$ and both can affect the Bragg peak intensity. To properly normalize the results, we employ the diffraction data described in Section 2.1.1 as a reference. From Equation 1, the Bragg peak intensity (denoted as $I_{r=0}$ ) of films deposited in a single pass with least air exposure (i.e., at $r=0 \mathrm{~mm}$ ) can be calculated for any thickness. This provides a deterministic link between the process conditions and the film structure.

The pentacene (001) Bragg peak intensity is measured along the $\boldsymbol{r}$-axis of a line deposit, denoted as $I_{r}$ and plotted in Figure 3c. $I_{\boldsymbol{r}}$ was measured along the $\boldsymbol{r}$-axis during the synchrotron experiments. The thickness values at each of the locations are known (Figure 1d) and are labeled individually in Figure 3c. Based on this information, we can quantitatively compare the measured film crystallinity via XRD in the "wing" region (Figure 3c), where moisture and oxygen in air can affect the crystallinity, to the expected values for the shielded region $(r=0 \mathrm{~mm})$ where the guard flow suppresses ambient exposure. (Note that the leading and trailing edges of the deposit at $r=0 \mathrm{~mm}$ along the translation axis experience some degree of air exposure, albeit, less than the wing region at large values of $r$.) This is important in properly deconvoluting the simultaneous contributions of growth rate dependent structural disorder and oxidation in the wings of the deposit to understand the practical limits of the patterning technique with regard to the desired film (and device) electronic properties. As can be observed from Figure 3d, the crystallinity of the film beyond the shielded zone $(r>$ $0.45 \mathrm{~mm}$ ) is reduced. An empirical curve is fit to the data and has a functional form of:

$$
I_{r} / I_{r=0}=\mathrm{A}_{1}+\left(\mathrm{A}_{2}-\mathrm{A}_{1}\right) /\left(1+\left(r / r_{\mathrm{a}}\right)^{p}\right)
$$

where $\mathrm{A}_{1}, \mathrm{~A}_{2}$, and $p$ are fitting constants, $r_{\mathrm{a}}$ is the point of inflection. $r_{\mathrm{a}}$ is affected by the nozzle geometry and the molecular flux distribution, while $p$ is mostly affected by the turbulent diffusion of air molecules (due to turbulent mixing of guard flow jet and ambient air) and the propensity of the semiconductor material to oxidize, although more detailed studies are needed to quantify these relationships. For the fit shown in Figure $3 \mathrm{~d}$, $r_{\mathrm{a}}=0.71 \pm 0.04 \mathrm{~mm}$ and $p=3.73 \pm 0.39$. This process-morphology relationship along the $\boldsymbol{r}$-axis can be further extended to include electronic properties of the films, as discussed in Section 2.3.

Note that for $r>1.5 \mathrm{~mm}$, where films are less than $12 \mathrm{~nm}$ thick, Equation 2 is invalid, due to a significant proportion of the very thin film having molecules with pentacene backbone axis parallel to the substrate, and the out of plane tilt angle 
(a)
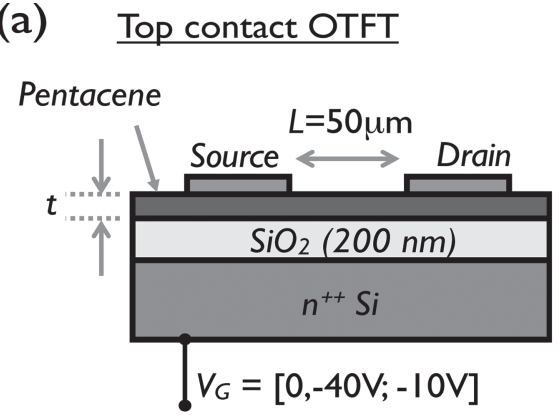

(c)

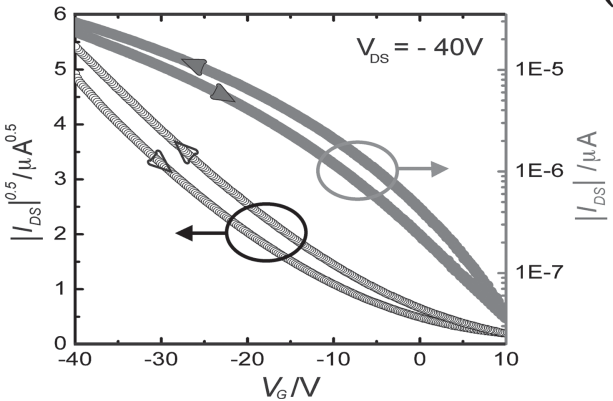

(b)

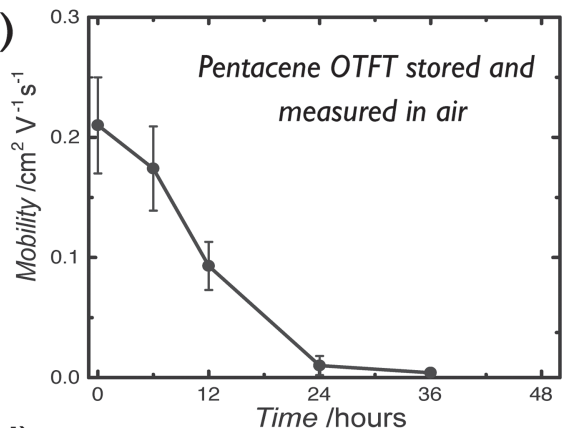

(d)

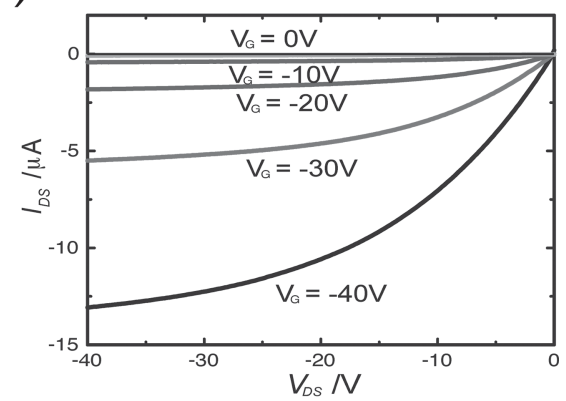

Figure 4. a) Schematic of a top contact pentacene TFT, fabricated on an n-type silicon wafer with a pre-deposited $200 \mathrm{~nm} \mathrm{SiO}_{2}$ gate dielectric. Pentacene film is deposited by GF-OVIP and source and drain electrodes are evaporated on top of the film using a shadow mask. b) Post deposition air exposure due to storage in ambient leads to degradation of pentacene films deposited. The lifetime of the devices that we fabricated is less than 48 hours after oxygen and moisture exposure. c,d) Standard current-voltage characteristics of our transistors, with $t=50 \mathrm{~nm}$.

being $28^{\circ}$, corresponding to a smaller lattice spacing than observed for thin-film phase. ${ }^{[34,35]}$

\subsection{Thin Film Transistor Performance of Pentacene Channels Deposited in Air}

Thin film transistors are fabricated with pentacene deposited in air by GF-OVJP at settings detailed in the Experimental section. The channel thickness is denoted by $t$, and its length $(L)$ and width $(W)$ are $50 \mu \mathrm{m}$ and $1 \mathrm{~mm}$, respectively. The device crosssection is shown schematically in Figure 4a. Control devices were deposited by VTE, as well as by GF-OVJP in a nitrogenpurged glovebox. Films of thickness between $15 \mathrm{~nm}$ and $80 \mathrm{~nm}$ were deposited in VTE at a deposition rate of $0.05 \mathrm{~nm} \mathrm{~s}^{-1}$. The in-plane, field-effect hole mobility was found to be highest between 25 and $35 \mathrm{~nm}$ film thickness. ${ }^{[36,37]}$ We estimate that the post-deposition degradation (Figure $4 \mathrm{~b}$ ) due to ambient exposure is gradual and minimal in comparison to the drop in TFT characteristics due to exposure during deposition. ${ }^{[38]}$

Pentacene channels grown by GF-OVJP in air exhibit the current-voltage characteristics shown in Figure 4c,d; hole field-effect mobility $\left(\mu_{\mathrm{sat}}\right)$, threshold voltage $\left(V_{\mathrm{T}}\right)$, subthreshold slope $(S)$, and On/Off ratio of current (On/Off) are all plotted in Figure 5a-d to show the comparison between air-deposited and glovebox-deposited pentacene films. We expect that oxygen incorporation will be lowest in VTE-grown channels, while GF-OVJP of pentacene channels in the glovebox should result in comparable amount of oxidation to the VTE samples, but enhanced molecular ordering and thus higher mobility. Indeed, this is seen in our experiments (Figure 5). The $\mu_{\text {sat }}$ of the glovebox deposited channels is almost double that of the air-deposited devices and follows the same trend at the VTE deposited controls, with the highest $\mu_{\mathrm{sat}}=0.38 \mathrm{~cm}^{2} \mathrm{~V}^{-1} \mathrm{~s}^{-1}$ recorded at $40 \mathrm{~nm}$ maximum feature thickness. $V_{\mathrm{T}}$ also follows a similar trend, with the glovebox deposited films having a lower $V_{\mathrm{T}}$ than their air deposited counterparts. $\mu_{\mathrm{sat}}$ and $V_{\mathrm{T}}$ are worse for the air deposited films due to the reaction of the hot organic vapor with oxygen in the periphery of the jet during deposition. It is worth noting that $\mu_{\text {sat }}$ is $30 \%$ lower for in-air deposited than in-glovebox deposited TFTs due to ambient oxygen and moisture exposure of the pentancene molecules of the former during deposition. From Figure $4 \mathrm{~b}$ a $30 \%$ drop in mobility is observed after $10 \mathrm{~h}$ of storage in ambient of VTE films due to post-deposition air exposure. Exposure to ambient oxygen and moisture has a far greater effect on vapor phase pentacene molecules at elevated temperatures "during deposition", than on post-deposited pentancene solid thin films.

The On/Off current ratio of the glovebox deposited films is higher than that of the air deposited films due to a lower leakage current in the Off state. These results are consistent with the prevailing understanding of pentacene TFTs, where oxidation results in bulk traps that reduce $\mu_{\text {sat }}$, while doping the film and elevating the Off current (more specifically, the formation of endoperoxides and hydroxyl variants of pentacene which disrupt the $\pi-\pi$ cloud of pentacene and distort the crystallinity of the film, respectively). ${ }^{[39]}$ In the next section we look at the effect of pentacene degradation by these two mechanisms due to air exposure during deposition. 


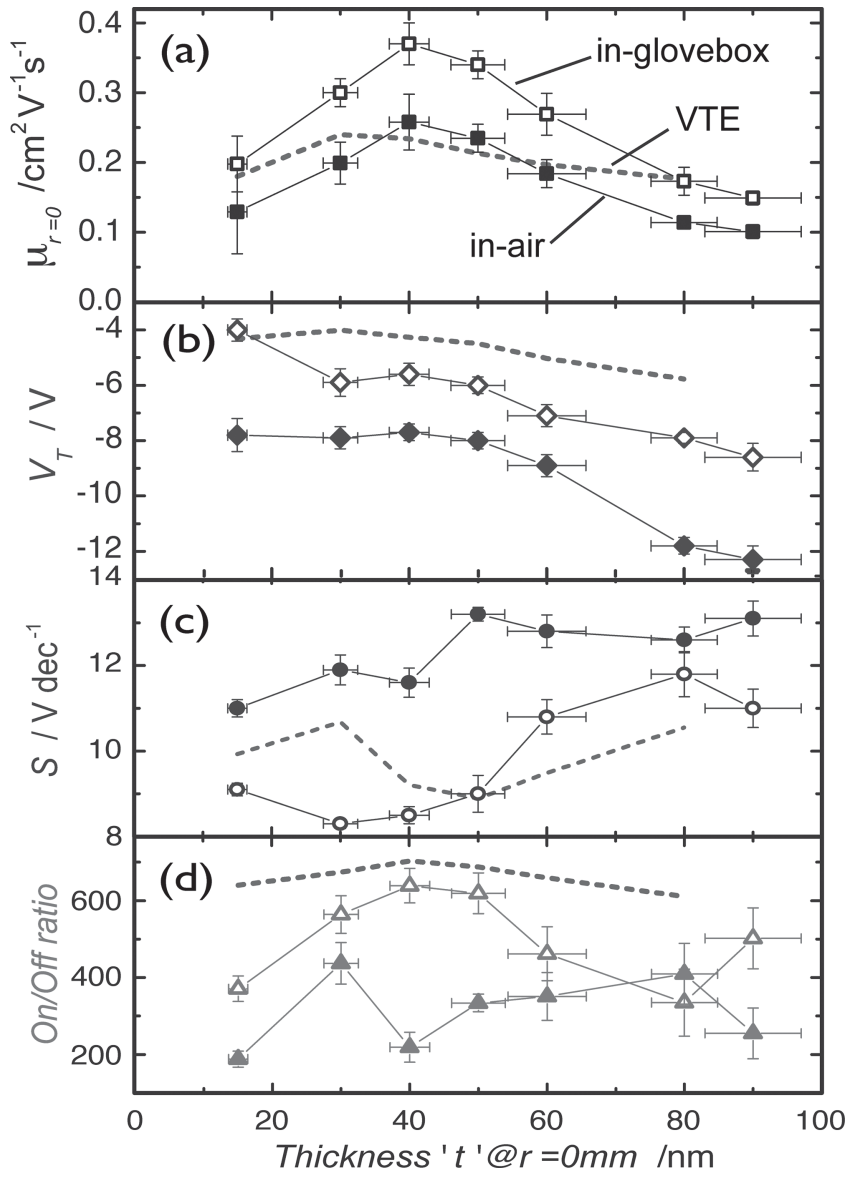

Figure 5. GF-OVJP deposited film characteristics: Filled symbols - In-air deposition; Open symbols: In-glovebox deposition; dashed grey line represents the TFT characteristics of VTE deposited films. Mobility, threshold voltage, sub-threshold slope and On/Off current ratio are measured at $r=0 \mathrm{~mm} . \mu_{\mathrm{r}=0}, \mathrm{~V}_{\mathrm{T}}$ peak around $t=40 \mathrm{~nm}$. In-glovebox to in-air difference in TFT characteristics indicates structural and electronic defects in air-exposed pentacene thin films.

\subsection{Degradation of Pentacene Electronic Properties from Air Exposure During Deposition: the Significance of Location Within the Deposited Feature}

The local deposition rate of a line deposited by GF-OVJP varies with $\boldsymbol{r}$. Figure $6 \mathrm{a}$ is a schematic of a line deposition process, showing the circular field of deposition area, along with the shielded zone and direction of deposition. From fluid dynamics simulation, we obtain the mole fraction of air at the boundary layer above the film as a function of $\boldsymbol{r}$, shown in Figure $3 \mathrm{~b}$ allowing for a calculation of the net air exposure of the pentacene film as a function of $r$. When fabricating TFTs, $1 \mathrm{~mm}$ wide gold contacts are deposited on top of the film, with the $50 \mu \mathrm{m}$ channel parallel to the $x$-axis and centered at different values of $\boldsymbol{r}$. Creating channels in this fashion allows us to correlate TFT performance with known amounts of oxygen and moisture exposure. In the "hot zone", at each value of $\boldsymbol{r}$, the total length of deposited pentacene film is $X_{\mathrm{r}}$, as shown in Figure 6a. Figure $6 \mathrm{~b}$ shows the average fraction of oxygen and moisture molecules (denoted as $\bar{N}_{r}^{\mathrm{O}_{2} \& \mathrm{H}_{2} \mathrm{O}}$ ) that interact with pentacene during deposition at each value of $\boldsymbol{r}$. This is obtained from the averaged weighted summation of the mole fraction of air along $x$-axis in the 'hot zone' at each value of $\boldsymbol{r}$ (denoted as $n_{r}^{\text {Air }}(x)$ ) shown in Figure 3b, given by

$$
\bar{N}_{\mathrm{r}}^{\mathrm{O}_{2} \& \mathrm{H}_{2} \mathrm{O}}=\frac{\int_{X_{\mathrm{r}}} \mathrm{n}_{r}^{\mathrm{Air}}(x) \partial x}{\int_{X_{\mathrm{r}}} \partial x} \times(0.2
$$

From Equation 3, we can calculate that the center, $r=0 \mathrm{~mm}$, has $50 \%$ total air exposure in comparison to the regions of the substrate that lay outside the field of deposition, while at $r=$ $1 \mathrm{~mm}$ the average air exposure increases to $65 \%$. Since even the centrally-located material in a line deposit contains elements of the wing region from the leading and trailing edges of the jet, the total air exposure fraction of $50 \%$ is not surprising. Quantitatively, about $11 \%$ of all molecules (including $\mathrm{N}_{2}$ from carrier gas, $\mathrm{N}_{2}$ from air, $\mathrm{O}_{2}, \mathrm{H}_{2} \mathrm{O}, \mathrm{CO}_{2}$, etc.) colliding with the pentacene molecules during deposition in the hot zone, at $r=0 \mathrm{~mm}$, are oxygen and moisture. Not all the oxygen and moisture interacting with pentacene molecules is incorporated into the deposited layer or reacts with hot organic vapor molecules to form chemical derivatives of pentacene. The values presented in Figure $6 \mathrm{~b}$ provide the absolute upper limit of defects/impurities in the deposited layer for the specific nozzle geometry and flow conditions used here.

To isolate the effect of ambient oxygen and moisture interaction with pentacene in the vapor and in the solid film during deposition, we fabricate thin film transistors at $r=0,0.4,0.6$, 1.0 and $1.5 \mathrm{~mm}$ from the center of the pentacene line deposits, both in air and in the glovebox. The source and drain contacts are deposited through a shadow mask such that the channel width is aligned to the direction of nozzle motion, $r$ away from the center, as shown in the Figure $6 c$ inset. The only difference between $\mu_{\mathrm{r}}$ and $\mu_{\mathrm{r}}^{\mathrm{G}}$ stems from the exposure of the air deposited samples to the ambient during deposition. The ratio of field-effect hole mobility values, measured in the saturation regime, for air deposited and glovebox deposited films, $\mu_{\mathrm{r}} / \mu_{\mathrm{r}}^{\mathrm{G}}$, at the $\boldsymbol{r}$-axis distances mentioned above for peak line thickness of $15 \mathrm{~nm}$ to $90 \mathrm{~nm}$ is plotted in Figure 6c.

$\mu_{\mathrm{r}} / \mu_{\mathrm{r}}^{\mathrm{G}}=\mathrm{A}_{4}+\left(\mathrm{A}_{4}-\mathrm{A}_{3}\right) /\left(1+\left(r / r_{\mathrm{a}}\right)^{p}\right)$

The dashed line fitted to the experimental data follows Equation 4 , with $r_{\mathrm{a}}=0.76 \pm 0.05 \mathrm{~mm}$ and $p=3.45 \pm 0.51$. The trend seen in this plot of $\mu_{\mathrm{r}} / \mu_{\mathrm{r}}^{\mathrm{G}}$ versus $r$ is consistent with the results obtained for the ratio of diffraction intensity of crystallinity along $r$, plotted in Figure $3 \mathrm{~d}$ and fit to Equation 2. This result is nearly identical to that in Section 2.1.2 for fitting X-ray diffraction peaks along $r$, and implies that the predominant influence on pentacene morphology degradation in the wing region, as observed in Figure 3d, comes from air exposure during deposition. The trend is also inversely proportional to the net air exposure of the film recorded in Figure 6b. Hence, we can relate the degree of air exposure during deposition to the film's morphology and electronic properties, recording a drop of $40 \%$ for both the crystallinity and charge mobility with position away from the center of the deposit along the $r$-axis. 
(a)

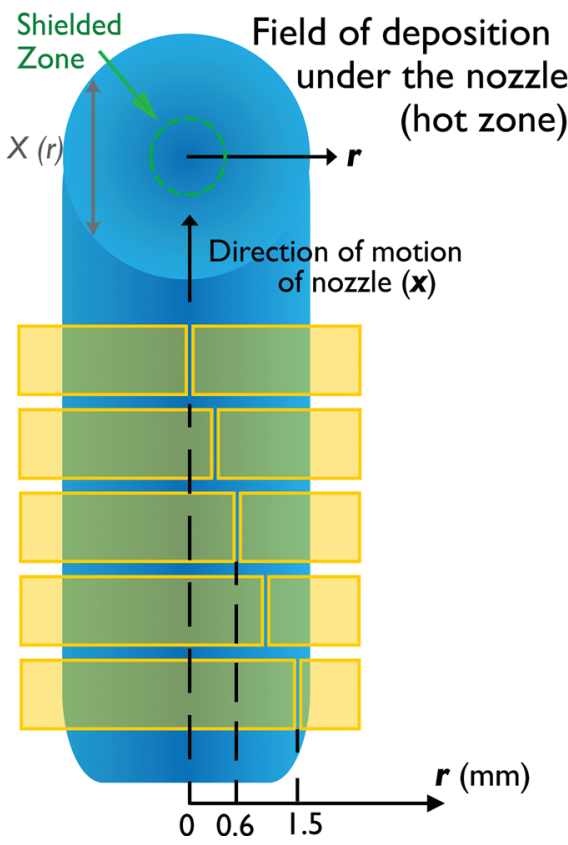

(b)

(c)

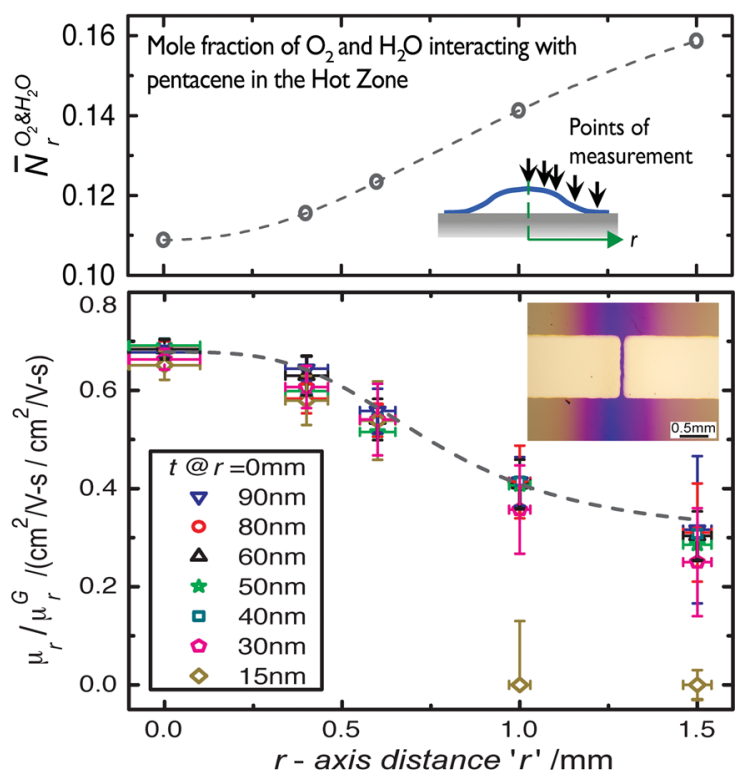

Figure 6. a) Top-view schematic of how the transistor channel is formed (i.e., source/drain gold contacts deposited by thermal evaporation through shadow masks, carefully aligned to center the channel along $r$ ); the channel thus contains material from both the shielded and the "wing" regions. The average mole fraction of oxygen and moisture that pentacene molecules interact with during deposition in the hot zone at each value of $r$ is plotted in (b). The dashed line is a curve fit to the data. c) Plot of the ratio of mobility of the films deposited in air to those deposited in the glovebox. The inset shows a micrograph of the TFT channel aligned on a line deposit.

In principle, any pattern can be generated by translating the nozzle over the substrate along a predefined path. The processstructure-property relationship obtained from the above study can be extended to predict the performance of these complex patterns in devices, since this relationship is independent of the nozzle, substrate relative motion which is the primary parameter used for drawing patterns. For example, rastering the nozzle over the substrates enables us to grow large area deposits of uniform thickness, as shown in Figure 1e. However, since the large area deposit thickness profile is an accumulation of thicknesses of line deposits uniformly spaced by $0.1 \mathrm{~mm}$, the total thickness of the deposit is given by $T=t_{r=0}+$ $2 \sum t_{\mathrm{r}}$. For total thickness in the thin film regime, $T<100 \mathrm{~nm}$, the influence of air exposure on the electronic properties can be averaged for the large area deposit from the known dependence of $\mu_{\mathrm{r}}$ versus $r$ of the line deposit. Thus the average mobility of a large area deposited in air by GF-OVJP can be estimated by:

$\mu_{T}=\frac{\mu_{t, r=0}+2 \sum\left(\mu_{r} t\right)}{t_{r=0}+2 \sum t}$

Using Equation 5 and extracting $\mu_{\mathrm{r}=0}$ and $\mu_{\mathrm{r}}$ from Figure $6 \mathrm{c}$, we predict $\mu_{\mathrm{T}}$ (for $T=50 \mathrm{~nm}$ ) to have a value of $0.21 \mathrm{~cm}^{2} \mathrm{~V}^{-1} \mathrm{~s}^{-1}$. Remarkably, the field-effect mobility for a separately printed, large-area deposit of total thickness of $50 \mathrm{~nm}$ is measured to be $0.21 \pm 0.06 \mathrm{~cm}^{2} \mathrm{~V}^{-1} \mathrm{~s}^{-1}$, and falls within the confidence interval of the predicted value. Thus, we can extend the process-structure-property relationship shown herein to accurately predict the performance of pentacene thin films in any pattern deposited in air using GF-OVJP.

\section{Conclusions}

To address a growing need to link process, structure, and properties in a predictive manner in the rapidly evolving area of organic semiconductors, we examine in detail pentacene thinfilm deposition by GF-OVJP, which enables additive patterning at high throughput of electronic device-quality organic semiconductor thin films in air. The guard flow shields the organic vapor-rich jet from exposure to ambient oxygen and moisture up to a certain distance along the $r$-axis. Outside this shielded zone, ambient air penetrates the boundary layer and interacts with the molecular film, disrupting its crystallinity and introducing trap states in the electronic band of the material. We use rigorous gas dynamics simulation to calculate the mole fraction of air molecules in the region outside the shielded zone, and relate that to the degree of disruption to molecular order in the film obtained by synchrotron-based XRD and by analyzing field-effect mobility measurements. From these correlations, we infer that some incorporation of air occurs in the deposited film during growth, resulting in a $40 \%$ drop in the crystallinity and field-effect mobility, which follow an identical trend across the radial axis. The interplay between defect incorporation, crystallinity and mobility is critical when printing complex patterns, as the effects of air exposure in the wings of the deposit profile have a cumulative effect on overall performance. We show conclusively that such effects can be accurately modeled for large area deposits, with the predicted field-effect mobility matching the measured values. These findings elucidate the relationship between deposition conditions, pattern dimensions, film morphology, and resulting device properties. They form a solid 
foundation for subsequent simulation-driven design of film deposition apparatus and process, in order to improve pattern resolution and device performance in organic electronics.

\section{Experimental Section}

GF-OVJP Apparatus and Deposition Conditions: The GF-OVJP nozzle used in this study was constructed from two coaxial glass tubes of 0.5 " and 0.75 " outer diameters, fused at one end and pulled at the other to form concentric nozzle tips of $200 \mu \mathrm{m}$ and $700 \mu \mathrm{m}$ internal diameters. The inert carrier gas and evaporated organic vapor is transported through the inner tube. The annular channel for the guard jet was found to be $100 \mu \mathrm{m}$ wide and angled at $\alpha \sim 15^{\circ}$ from the nozzle axis. The nozzle was cleaned with solvents, ${ }^{[29]}$ dried and wrapped with 36-gauge heavy insulated tape heater (Omega Engineering, Inc.) with a power density of $8.6 \mathrm{~W} \cdot \mathrm{in}^{-2}$. The heating tape leads were connected to a temperature controller (Digi-Sense Benchtop temperature controller, Cole-Palmer Instruments Co.) and a 1/16" K-type thermocouple was used to maintain the temperature of the source. The source consisted of $0.15 \mathrm{~g}$ of pentacene powder (99\% purity, Luminescence Technology Corp.) sandwiched between quartz wool and placed in the heated source section of the inner tube. The carrier gas and guard jet flow rates were maintained using mass flow controllers (C100 MFC, Sierra Instruments). The nozzle axis is held perpendicular to the substrate, and its motion is achieved by a computer-controlled X-Y-Z positioning stage (PT3-Z8 motorized translation stage, Thorlabs).

The process parameters, as shown in Figure 1, are nozzle-substrate separation, $s$, evaporation/sublimation zone temperature, $T_{\mathrm{Ev}}$, substrate temperature, $T_{S}$, carrier gas flow rate, $Q_{\mathrm{CC}}$ and guard flow rate, $Q_{\mathrm{GF}}$. Powdered pentacene is loaded in the sublimation zone and heated to $T_{\mathrm{Ev}}=260.0 \pm 0.5^{\circ} \mathrm{C} . T_{\mathrm{S}}$ is maintained at room temperature while the $Q_{\mathrm{CG}}$, $Q_{\mathrm{CF}}$, and $\mathrm{s}$ held constant at $200 \mathrm{sccm}, 600 \mathrm{sccm}$, and $1 \mathrm{~mm}$ respectively.

Computational Fluid Dynamics Modeling of Jet Flow and Organic Material Deposition: To fully understand the hydrodynamic interaction of guard flow jet and the primary jet carrying organic vapor, and the subsequent film growth and incorporation of ambient air into film, a complete computational fluid-dynamic modeling by FLUENT 6.3.26 software is utilized. Using Navier-Stokes equations with a standard $k-\varepsilon$ turbulence model in the software coupled with estimated fitting parameters, multicomponent diffusion and species transport describing organic vapor transport and adsorption, a full map of flow and species distribution characteristics were obtained. ${ }^{[27]}$

Synchrotron X-Ray Diffraction Measurement Set Up: X-ray diffraction experiments were conducted at the beamline X21 (wiggler source) of the National Synchrotron Light Source (NSLS) at Brookhaven National Laboratory, and at the beamline 33-IDD (undulator source) of the Advanced Photon Source (APS) at Argonne National Laboratory. The data given in Figure 2 was measured with $10.0 \mathrm{keV}$ monochromatic $\mathrm{X}$-ray beam at beamline X21 of NSLS. For the data given in Figure $3 \mathrm{c}, \mathrm{d}$, highly focused undulator beam (beam size $\approx 60 \mu \mathrm{m} \times 60 \mu \mathrm{m}, 10 \mathrm{keV}$ $X$-ray energy) at the beamline 33-IDD of APS was employed. For all measurements, standard $\theta-2 \theta$ scans were taken in the vertical scattering plane with a PILATUS $100 \mathrm{~K}$ pixel detector. Pentacene films were grown using GF-OVJP in air on $5 \mathrm{~cm} \times 5 \mathrm{~cm}$ bare silicon substrates, which were cleaned as described below.

Pentacene Thin Film Transistor Fabrication and Testing: Substrates used in this study were $2.5 \mathrm{~cm} \times 2.5 \mathrm{~cm}$ doped silicon wafers with a $200 \mathrm{~nm}$ thick oxide layer. These were cleaned by ultrasonication in detergent solution and deionized water followed by heated acetone, trichloroethylene, and isopropanol for $10 \mathrm{~min}$ each. Substrates were then placed in boiling isopropanol for $5 \mathrm{~min}$, dried in pure nitrogen gas prior to pentacene film deposition. Control devices were fabricated with films deposited by thermal evaporation in a $10^{-6}$ Torr vacuum chamber (Angstrom Engineering Inc.). Pentacene patterned by GF-OVJP was done in air and in an LC Technology Solutions Inc. glovebox purged with 99.99\% pure $\mathrm{N}_{2}$. The gold source-drain electrodes were deposited on the patterned pentacene film surface by shadow mask evaporation. The channel had a thickness, $t$, with $L=50 \mu \mathrm{m}$ and $W=1 \mathrm{~mm}$. The channel was carefully aligned at the exact location along the $r$-axis of the deposit using a Zeiss Axio Scope.A1 upright microscope. Five transistors were fabricated on each pentacene line in order to obtain the error bars recorded in this work. The organic TFT electrical properties were tested in ambient using an Agilent 4156B semiconductor parameter analyzer.

\section{Acknowledgments}

The authors MS and SB thanks the Air Force Office of Scientific Research (AFOSR) for its Presidential Early Career Award for Scientists and Engineers (PECASE), Award No. FA9550-09-1-0109, for supporting the development of the GF-OVJP technique, analysis of compressible hydrodynamics, and its application to deposition organic TFT materials and devices performed in this work. MS, RC, SB, YY and CMS acknowledge funding from Center for Solar and Thermal Energy Conversion, and Energy Frontier Research Center supported by the US Department of Energy (DOE), Office of Basic Energy Sciences (Award No. DE-SC0000957) for supporting the high resolution XRD experiments and data analysis performed at the Brookhaven National Laboratory (BNL), as well as the analytical work on predicting field effect mobility as a function of deposition conditions. BNL NSLS experiments were also supported by the U.S. Department of Energy (Contract No. DE-FG0206ER46273, PI: RC and Contract No. DE-AC02-98CH10886) while the use of APS at Argonne National Laboratory was supported by the U.S. Department of Energy, Office of Science, Office of Basic Energy Sciences, under Contract No. DE-AC02-06CH11357. RLH was supported by grant No. DE-FG02-07ER46380 from the U.S. Department of Energy, Office of Basic Energy Sciences, Division of Materials Sciences and Engineering. The authors thank N. Senabulya for her help in synchrotron data acquisition. Excellent beamline support by C. Nelson and S. LaMarra (NSLS-X21), Z. Zhang (APS-33IDD), and the staff of the NSLS and the APS is gratefully acknowledged.

Received: November 26, 2013

Revised: January 11, 2014

Published online: March 3, 2014

[1] National Science and Technology Council, Office of Science and Technology Policy, Materials Genome Initiative for Global Competitiveness June 2011.

[2] S. R. Forrest, Nature 2004, 428, 911.

[3] S. Biswas, O. Shalev, M. Shtein, Annu. Rev. Chem. Biomol. Eng. 2013, 4, 289.

[4] Y. S. Tyan, J. Photonics Energy 2011, 1, 011009.

[5] B. P. Rand, J. Genoe, P. Heremans, J. Poortmans, Prog. Photovoltaics: Res. Appl. 2007, 15, 659.

[6] M. Kitamura, Y. Arakawa, J. Phys.: Condens. Matter 2008, 20, 184011.

[7] G. Horowitz, J. Mater. Res. 2004, 19, 1946.

[8] J. Smith, W. Zhang, R. Sougrat, K. Zhao, R. Li, D. Cha, A. Amassian, M. Heeney, I. McCulloch, T. D. Anthopoulos, Adv. Mater. 2012, 24, 2441.

[9] H. E. Katz, Chem. Mater. 2004, 16, 4748.

[10] D. J. Gundlach, Y. Y. Lin, T. N. Jackson, S. F. Nelson, D. G. Schlom, IEEE Electron Device Lett. 1997, 18, 87.

[11] C. C. Mattheus, G. A. de Wijs, R. A. de Groot, T. T. M. Palstra, J. Am. Chem. Soc. 2003, 125, 6323.

[12] C. D. Dimitrakopoulos, P. R. L. Malenfant, Adv. Mater. 2002, 14, 99.

[13] W.-Y. Chou, H.-L. Cheng, Adv. Funct. Mater. 2004, 14, 811.

[14] M. Shtein, J. Mapel, J. B. Benzinger, S. R. Forrest, Appl. Phys. Lett. 2002, 81, 268

[15] F. J. Meyer zu Heringdorf, M. C. Reuter, R. M. Tromp, Nature 2001, 412, 517.

[16] B. Chwang, C. D. Frisbie, J. Phys. Chem. B 2000, 104, 12202. 
[17] Y.-Y. Lin, D. J. Gundlach, S. F. Nelson, T. N. Jackson, IEEE Trans. Electron Devices 1997, 44, 1325.

[18] S. Kobayashi, T. Nishikawa, T. Takenobu, S. Mori, T. Shimoda, T. Mitani, H. Shimotani, N. Yoshimoto, S. Ogawa, Y. Iwasa, Nat. Mater. 2004, 3, 317.

[19] C. W. Chu, S. H. Li, C. W. Chen, V. Shrotriya, Y. Yang, Appl. Phys. Lett. 2005, 87, 193508

[20] J. Puigdollers, C. Voz, A. Orpella, I. Martin, M. Vetter, R. Alcubilla, Thin Solid Films 2003, 427, 367.

[21] M. Shtein, H. F. Gossenberger, J. B. Benzinger, S. R. Forrest, J. Appl. Phys. 2001, 89, 1470.

[22] C. Cali, V. Daneu, A. Orioli, S. Riva-Sanseverino, Appl. Opt. 1976, 15, 1327.

[23] J. Y. Lee, S. T. Lee, Adv. Mater. 2004, 16, 51.

[24] J. Chen, V. Leblanc, S. H. Kang, P. J. Benning, D. Schut, M. A. Baldo, M. A. Schmidt, V. Bulovic, Adv. Funct. Mater. 2007, 17, 2722.

[25] M. Shtein, P. Peumans, J. B. Benziger, S. R. Forrest, J. Appl. Phys. 2004, 96, 4500.

[26] C. Yun, J. Choi, H. W. Kang, M. Kim, H. Moon, H. J. Sung, S. Yoo, Adv. Mater. 2012, 24, 2857.

[27] S. Biswas, K. A. Luck, M. Shtein, Org. Electron. 2012, 13, 2905.
[28] H. Aziz, Z. Popovic, S. Xie, A.-M. Hor, N.-X. Hu, C. Tripp, G. Xu, Appl. Phys. Lett. 1998, 72, 756.

[29] S. Biswas, K. P. Pipe, M. Shtein, Appl. Phys. Lett. 2010, 96, 263301.

[30] O. Shalev, M. Shtein, Org. Electron. 2013, 14, 94.

[31] M. Shtein, P. Peumans, J. B. Benziger, S. R. Forrest, J. Appl. Phys. 2003, 93, 4005.

[32] Y. R. Sun, M. Shtein, S. R. Forrest, Appl. Phys. Lett. 2005, 86, 113504.

[33] B. E. Warren, X-Ray Diffraction, Dover Publications, Inc., New York, NY, USA 1990, Ch. 3.

[34] S. Söhnchen, S. Lukas, G. Witte, J. Chem. Phys. 2004, 121, 525.

[35] F. Zheng, B.-N. Park, S. Seo, P. G. Evans, F. J. Himpsel, J. Chem. Phys. 2007, 126, 154702.

[36] R. Ruiz, A. Papadimitratos, A. C. Mayer, G. G. Malliaras, Adv. Mater. 2005, 17, 1795.

[37] J. E. Northrup, M. L. Chabinyc, Phys. Rev. B 2003, 68, 041202.

[38] When stored in air, the films degrade in time, as plotted for $t=40 \mathrm{~nm}$ deposited by VTE in Figure 4b. This is suspected to be due to reaction of pentacene with ambient oxygen and moisture resulting in the formation of endoperoxide, giving rise to trap states in the electronic gap and hydroxyl impurities, which distort the crystallinity of the thin-film.

[39] F. De Angelis, M. Gaspari, A. Procopio, G. Cuda, E. Di Fabrizio, Chem. Phys. Lett. 2009, 468, 193. 